\title{
Characterization of Localized Elemental Composition of Core-Shell Pd/C Catalysts by FESEM/EDS and STEM/EDS
}

\author{
Y.C. Wang ${ }^{1 *}$ and M. Kulzick ${ }^{1}$ \\ 1. BP Amoco Chemical Co, Naperville, IL, USA. \\ * Corresponding author: youngchung.wang@bp.com
}

Commercial $0.5 \% \mathrm{Pd} / \mathrm{C}$ catalysts are used for the production of purified terephthalic acid (PTA). This material has been widely studied by a range of physical techniques and by activity tests performed in conditions strictly similar to those of industrial operation (1). These catalysts show a strongly external $\mathrm{Pd}$ distribution (eggshell type), pointing to high $\mathrm{Pd}$ concentrations in the outer layers of the carrier. $\mathrm{Pd}$ is located in the structure on the exterior surface of the carbon, in macropores penetrating into the carbon, and in the micropore structure of the carbon.

Understanding the location and concentration gradients for the $\mathrm{Pd}$ in these catalysts is critical to understanding catalyst performance. Due to the complex structure of coconut shell carbon, accurate understanding of the localized Pd concentration in the carbon granules is complicated. We will present methods for the evaluation of these concentration differences using SEM and STEM-EDS (2).

To map the Pd distribution in the overall carbon granules FESEM-EDS was used at low magnification. Catalyst granules are cross-sectioned by cutting a few granules at right angles to the granule axis with a single edge steel razor blade. Another cut is made at the other end of the granule, so granules will stand vertically enabling encapsulation by epoxy molding compound. These embedded samples are then polished allowing the localization of $\mathrm{Pd}$ in the coconut shell granule to be imaged and the composition determined relative to the exterior surface of the granule.

The specimen is analyzed in the FE-SEM with standard detectors for secondary and backscatter electrons using appropriate operating conditions and setup parameters. To map the composition of the entire granule low 100x magnification is typically used at a $100 \mathrm{sec}$ per frame rate. To determine the concentration profile of the granule, an area large enough to accommodate 8-10 analysis areas of 100 $\mathrm{x} 100$ microns is found and an SEM-EDS map of the entire region obtained. Figure 1 is an example of such a composition map for a commercial $\mathrm{Pd} / \mathrm{C}$ catalyst.

To determine the quantitative element concentrations, the granule must be divided into an array of equivalent analysis areas. Due to local heterogeneity in the materials, it is critical to analyze equivalent areas and to use large enough areas that local differences can be randomized. This is typically done for a granule quadrant as shown in Figure 2. Calculate the quantitative SEM-EDS results for each 100 by 100 micron region in the array. These analysis regions are typically spaced at 250 micron increments from each other. The data can be reported on the analyzed image or exported to a spreadsheet for further analysis. This is repeated for all four quadrant areas of the granule. A sufficient number of granules are analyzed to form a statistically relevant average composition map. To obtain an average radial distribution of concentration from the 
edge to the center of the granule, results from analysis zones of equivalent distance from the granule exterior are averaged and plotted as a function of distance from granule edge (Figure 3).

This procedure can be repeated at higher magnification to map regions with higher Pd localized concentration gradients such as near the surface of the Pd granule. A similar procedure is followed with analysis of areas of equivalent area at known distances from the edge of the granule. These can be averaged as a function of distance from the edge to form a representation of the average radial concentration distribution in the region of interest.

A higher localization of the Pd distribution can be obtained by STEM-EDS. A thin microtomed crosssection of the epoxy mounted granule including the exterior edge is obtained and analyzed as shown in Figure 4. The high localization of the Pd on the granule surface is evident. Using a method similar to that described for the SEM-EDS data, this distribution of the elemental composition from the granule exterior to the interior is obtained. In the STEM-EDS image this can be related to the macro and micropore structure of the carbon.

References:

[1] S Hannemann et al., Applied Surface Science 252 (2006), p. 7862.

[2] PL Gai and ED Boyes, Electron Microscopy in Heterogeneous Catalysis, (Inst. of Physics Publishing, Bristol, Philadelphia).
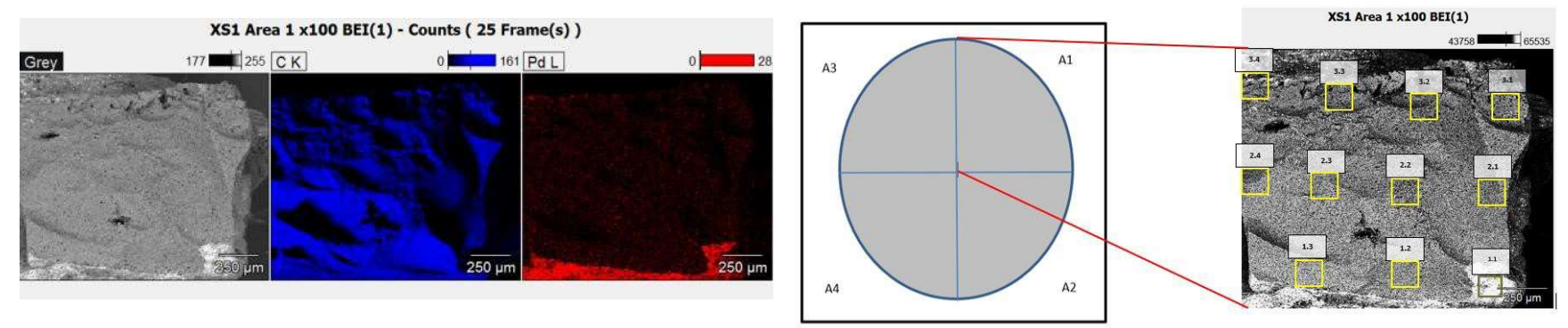

Figure 1. Example of XS-Map with C, and Pd

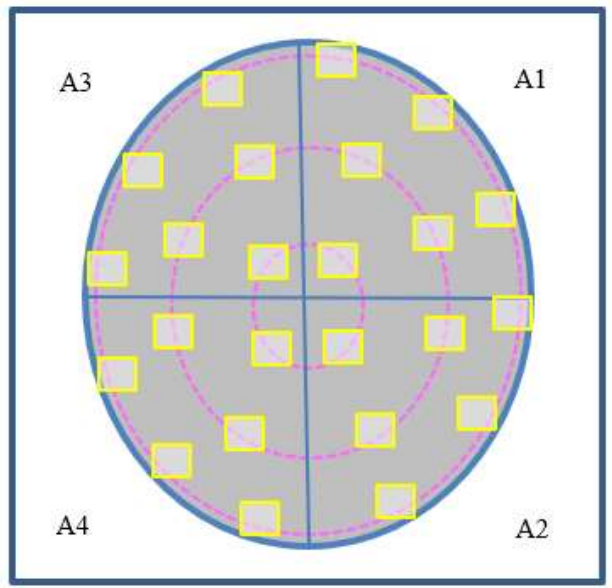

Figure 3. The concentric dotted lines indicate the possibility to create an average radial distribution graph of the analyzed
Figure 2. Example of an image cross section area and image with measurement boxes.

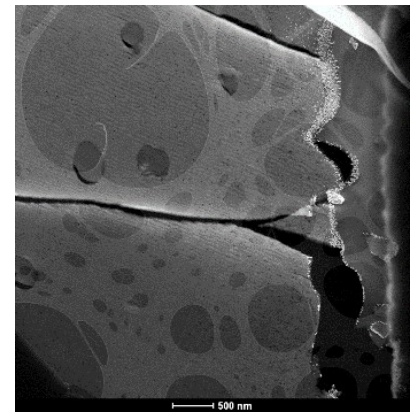

STEM-HAADF image ( $z$ contrast) $\mathrm{Pd}$ particles with bright contrast

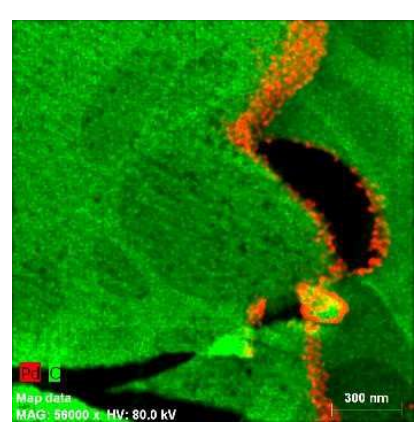

STEM-EDS map

$\mathrm{Pd}$ (in red) is detected in carbon micropores
Figure 4. A thin microtomed cross-section of the epoxy mounted granule including the exterior edge is obtained and analyzed. 